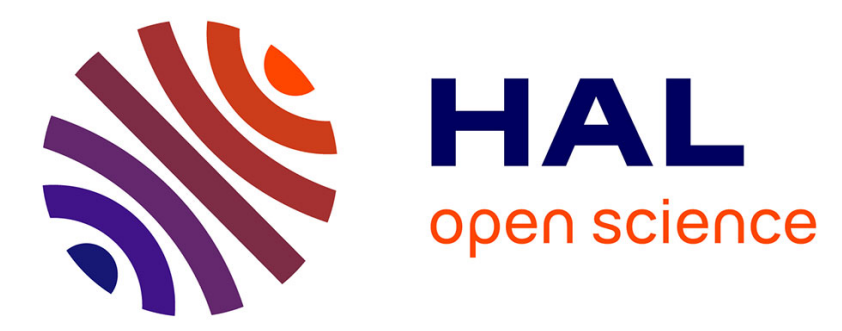

\title{
Fast Digital Filtering of Spectrometric Data for Pile-up Correction
}

\author{
T Trigano, Eric Barat, Thomas Dautremer, Thierry Montagu
}

\section{To cite this version:}

T Trigano, Eric Barat, Thomas Dautremer, Thierry Montagu. Fast Digital Filtering of Spectrometric Data for Pile-up Correction. IEEE Signal Processing Letters, 2014, 22 (7), pp.973-977. 10.1109/LSP.2014.2377352 . cea-01158652

\section{HAL Id: cea-01158652 https://hal-cea.archives-ouvertes.fr/cea-01158652}

Submitted on 1 Jun 2015

HAL is a multi-disciplinary open access archive for the deposit and dissemination of scientific research documents, whether they are published or not. The documents may come from teaching and research institutions in France or abroad, or from public or private research centers.
L'archive ouverte pluridisciplinaire HAL, est destinée au dépôt et à la diffusion de documents scientifiques de niveau recherche, publiés ou non, émanant des établissements d'enseignement et de recherche français ou étrangers, des laboratoires publics ou privés. 


\title{
Fast Digital Filtering of Spectrometric Data for Pile-up Correction
}

\author{
T. Trigano, Member, IEEE, E. Barat, T. Dautremer and T. Montagu
}

\begin{abstract}
This paper considers a problem stemming from the analysis of spectrometric data. When performing experiments on highly radioactive matter, electrical pulses recorded by the spectrometer tend to overlap, thus yielding severe distortions when computing the histogram of the pulses' energies. In this paper, we propose a fast recursive algorithm which estimates efficiently this histogram from measurements of the duration and energies of overlapping pulses. Its good performances are shown both on simulations and real data. Furthermore, its lower algorithmic complexity makes it more fitting for real-time implementation.
\end{abstract}

Index Terms-Nonlinear inverse problem, digital signal processing, signal restoration and enhancement, pile-up correction

\section{INTRODUCTION}

$\mathbf{T}$ HE main objectives of $\gamma$ spectrometry are the identification of unknown radioactive sources and the estimation of their activities, determined by the information stemming from photons emitted at random times. A digital spectrometry apparatus converts the energy of a photon impinging on a detector into a electrical pulse of finite duration, whose associated energy (in that case, the sum of all samples of the pulse) is quantized and recorded. At the end of the experiment, the energy distribution is estimated by means of a histogram, which is called the energy spectrum and provides information on the emitting source. It is therefore relevant in that framework to consider an energy spectrum as a discrete probability distribution to be estimated in a nonparametric setting.

In practice, the inter-arrival times between photons can be shorter than the durations of generated electrical pulses after amplification, thus creating clusters of pulses, known as pile-ups. An example of pile-ups occurring on a real signal is presented in Figure 1a. This also causes a distortion of the energy spectrum that becomes more severe as the activity increases. More specifically, standard spectrum analyzers consider pile-ups as single electrical pulses, and record the sum of the pulses' individual energies instead of each energy separately. Therefore, fake sum peaks in the energy spectrum and a distortion of its continuous part, called the Compton continuum, can be observed. Both distortions make the final identification of the source more difficult for the practitioner.

Copyright (c) 2012 IEEE. Personal use of this material is permitted. However, permission to use this material for any other purposes must be obtained from the IEEE by sending a request to pub-permissions@ieee.org

T. Trigano is with the Department of Electrical Engineering, Shamoon College of Engineering, Ashdod, Israel. E. Barat, T. Dautremer and T. Montagu are with CEA, LIST, F-91191 Gif sur Yvette, France.

Manuscript received May 13, 2015.
Figure $1 \mathrm{~b}$ illustrates such distortion for a simple radioactive element, Cesium 137.

Recent pile-up correction techniques include DSP real time compensation [2] and phase correlation coupled with maximum likelihood estimation [3]. These methods are based on assumptions regarding the pulse shape, and perform well under good signal to noise ratios. Also recently, the authors presented in [4] an adaptation of the formula appearing in [1], and derived from it an algorithm based on the numerical inversion of a non-linear equation. Unfortunately, the method presented in [4] has high algorithmic complexity, which makes it unsuitable for real-time implementation.

The aim of this paper is to propose a fast algorithm for pile-up correction, based on the inversion formula given in [4]. We first recall the mathematical model used as well as the inversion formula used in this framework. We then detail a fast algorithm to derive an estimate of the corrected energy spectrum. Eventually, we discuss the performances of the detailed approach, both on simulations and real data.

\section{Algorithm FOR Digital PILE-UP CORRECTION}

We recall in this section the mathematical model used and the inversion formula appearing in [4]. This pile-up correction formula can be seen as an adaptation of $|5|$ to the discrete case, and will be the basis of a new estimator of the energy distribution for discrete and quantified signals. In order to avoid cumbersome notations, we assume that the sampling and quantization periods are equal to 1 .

\section{A. Mathematical model and discrete pile-up correction for- mula}

The observed discrete-time signal is modeled as

$$
s[n]=\sum_{k=1}^{+\infty} \Phi_{k}\left[n-\left\lceil T_{k}\right\rceil\right],
$$

where $\left\{T_{k}, k \geq 1\right\}$ is the ordered sequence of the points of a homogeneous Poisson process with known intensity $\lambda$, $\Phi_{k}$ is the $k$-th recorded electrical pulse with duration $X_{k}$ and energy $Y_{k}$. Note that the term energy in this framework must be understood as the sum of the samples, that is $Y_{k}$ is the sum of the samples $s[n]$ between $\left\lceil T_{k}\right\rceil$ and $\left\lceil T_{k}\right\rceil+X_{k}$. We assume that $\left\{\left(X_{k}, Y_{k}\right), k \geq 1\right\}$ is a sequence of independent and identically distributed integer-valued discrete random variables, independent of $\left\{T_{k}, k \geq 1\right\}$, with finite expectation and common probability mass function $p_{X, Y}$. We also denote by $p_{Y}$ the marginal distribution of the $Y_{k}$ 's, which 


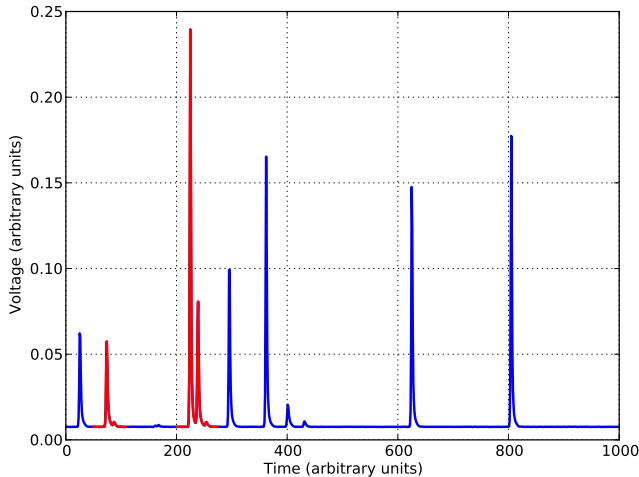

(a)

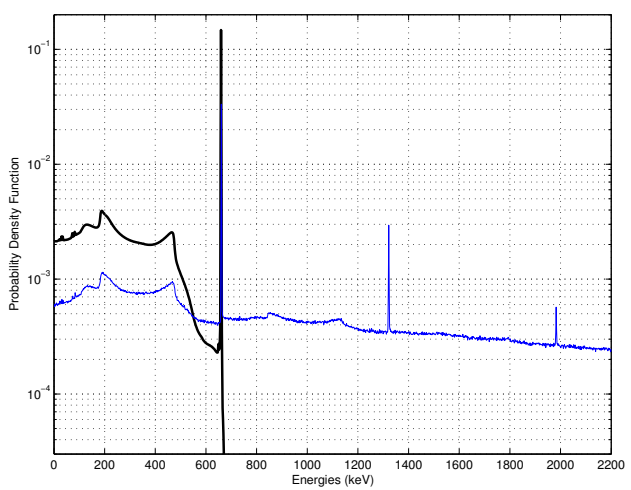

(b)

Fig. 1: Influence of the pile-up effect on a real signal from an high purity Germanium detector, with high signal-noise ratio. $\mathrm{T}$ (a) time domain, the pile-ups are displayed in red.; (b) Theoretical probability density function of the energy (black) and observed piled-up histogram at a counting rate $\lambda=10^{6}$ photons per second (blue line). We observe fake sum pikes at multiples of $667 \mathrm{keV}$ and a distortion of the Compton continuum. (Taken from [1]).

corresponds in practice to the ideal energy spectrum that we would estimate if we disposed of direct observations of $Y_{k}$. In practice, however, the shapes $\Phi_{k}$ are unknown, and neither $X_{k}$ nor $Y_{k}$ can be observed directly. Instead, the spectrometer device [6] derives the arrival times of the detector's busy periods $T_{k}^{\prime}$, their durations $X_{k}^{\prime}$ and their energies $Y_{k}^{\prime}$ from $s[n]$ :

$$
\begin{aligned}
T_{k}^{\prime} & =\min _{m \geq T_{k-1}^{\prime}}\{s[m]=0, s[m+1]>0\}, \\
X_{k}^{\prime} & =\min _{m \geq T_{k}^{\prime}}\{s[m]>0, s[m+1]=0\}, Y_{k}^{\prime}=\sum_{m=T_{k}^{\prime}}^{T_{k}^{\prime}+X_{k}^{\prime}} s[m] .
\end{aligned}
$$

It can be shown (see e.g. [5]), that the random variables $\left\{\left(X_{k}^{\prime}, Y_{k}^{\prime}\right), k \geq 1\right\}$ are also independent and identically distributed. The physical significance of $T_{k}^{\prime}, X_{k}^{\prime}, Y_{k}^{\prime}$ is summarized in Figure 2. We also denote by $p_{X^{\prime}, Y^{\prime}}$ the common probability mass distribution of the $\left\{\left(X_{k}^{\prime}, Y_{k}^{\prime}\right), k \geq 1\right\}$, and by $P_{X^{\prime}, Y^{\prime}}\left(z_{1}, z_{2}\right) \stackrel{\text { def }}{=} \sum_{m \geq 0} \sum_{n \geq 0} p_{X^{\prime}, Y^{\prime}}[n, m] z_{1}^{n} z_{2}^{m}$ its moment-generating function.

The problem to address in practice is to estimate $p_{Y}$ given a sample of observed durations and energies of busy periods $\left(X_{k}^{\prime}, Y_{k}^{\prime}\right), k=1 \ldots N$. The cornerstone of this estimation will be based on the following result:

Theorem 1 (pile-up correction formula [7], [5] ): Define $k[n, m] \stackrel{\text { def }}{=} \sum_{j=0}^{n} \sum_{i=0}^{n-j} p_{X, Y}[i, m]$, that is the doubly cumulative sum with respect to the first component of $p_{X, Y}$. Under the previous assumptions, we have for all $\left|z_{1}\right| \leq 1,\left|z_{2}\right| \leq 1$ :

$$
\begin{aligned}
\sum_{n \geq 0}(\exp & \left.\left(-\lambda n+\lambda \sum_{m \geq 0} k[n, m] z_{2}^{m}\right)\right) z_{1}^{n} \\
= & \frac{1}{1-\left(\mathrm{e}^{-\lambda} z_{1}+\left(1-\mathrm{e}^{-\lambda}\right) z_{1} P_{X^{\prime}, Y^{\prime}}\left(z_{1}, z_{2}\right)\right)} .
\end{aligned}
$$

Due to the definition of $k[n, m]$, we find that

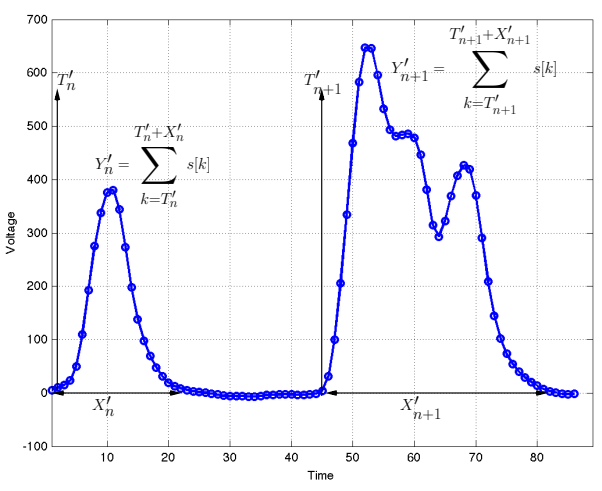

Fig. 2: Summary of the notations on a real signal $s[n]$ (the sampling points are circled). In this example, the first busy period is actually a single pulse, whereas the second is a pileup of three pulses.

and since $k[0, m]=k[-1, m]=k[-2, m]=0$ :

$$
p_{Y}[m]=\sum_{n \geq 0} p_{X, Y}[n, m]=\lim _{n \rightarrow \infty}(k[n, m]-k[n-1, m]) .
$$

Thus, (2) states that a nonlinear relation exists between a functional of $p_{Y}$, from which we have no direct observations but wish to estimate, and a functional of $p_{X^{\prime}, Y^{\prime}}$ from which observations can be obtained experimentally. Therefore, it is possible to estimate $p_{Y}$ by direct numerical inversion of (2), as in [7]. Though this method provides acceptable results, it may be noticed that the problem can be addressed more elegantly in the time domain, without explicit inversion.

\section{B. Fast estimation of the energy spectrum}

We now present a fast algorithm to obtain an estimate of $p_{Y}$. Denote by $Y\left(z_{1}, z_{2}\right)$ the Right Hand Side (RHS) of 22; since $Y\left(z_{1}, z_{2}\right)$ is analytic, we denote by $y[n, m]$ $(k[n, m]-k[n-1, m])-(k[n-1, m]-k[n-2, m])=p_{X, Y}[n, m]$. the terms of its power series expansion, that is $Y\left(z_{1}, z_{2}\right)=$ 
$\sum_{n>0} \sum_{m>0} y[n, m] z_{1}^{n} z_{2}^{m}$. Comparing $Y\left(z_{1}, z_{2}\right)$ to the Left Hand Side (LHS) of (2) yields

$$
\exp \left(-\lambda n+\lambda \sum_{m \geq 0} k[n, m] z_{2}^{m}\right)=\sum_{m \geq 0} y[n, m] z_{2}^{m},
$$

or equivalently by taking the log-derivative in $(3)$ with respect to $z_{2}$ :

$$
\begin{aligned}
\sum_{m \geq 0} \lambda k[n, m+1] & (m+1) z_{2}^{m} \\
= & \frac{\sum_{m \geq 0}(m+1) y[n, m+1] z_{2}^{m}}{\sum_{m \geq 0} y[n, m] z_{2}^{m}} .
\end{aligned}
$$

Rewriting (4) using a Cauchy product, we obtain

$$
\begin{aligned}
& \sum_{m \geq 0}(m+1) y[n, m+1] z_{2}^{m} \\
& =\sum_{m \geq 0}\left(\sum_{k=0}^{m} y[n, k] \lambda k[n, m-k+1](m-k+1)\right) z_{2}^{m} .
\end{aligned}
$$

Equating the terms of the powers series leads to

$$
\begin{aligned}
& (m+1) y[n, m+1]=\lambda(m+1) y[n, 0] k[n, m+1] \\
& +\sum_{k=1}^{m} y[n, k] \lambda k[n, m-k+1](m-k+1)
\end{aligned}
$$

which provides the recursive computation of $k[n, m]$ as a function of $y[n, m]$ :

$$
\begin{aligned}
k[n, m]=\frac{y[n, m]}{\lambda y[n, 0]} & \\
& \quad-\frac{1}{m} \sum_{k=1}^{m-1}(m-k) \frac{y[n, k]}{y[n, 0]} k[n, m-k] .
\end{aligned}
$$

On the other hand, by expanding the RHS of (2) into power series, we obtain an explicit recursive computation of $y$. Specifically, since

$$
\begin{aligned}
Y\left(z_{1}, z_{2}\right)- & \mathrm{e}^{-\lambda} z_{1} Y\left(z_{1}, z_{2}\right) \\
& -\left(1-\mathrm{e}^{-\lambda}\right) z_{1} P_{X^{\prime}, Y^{\prime}}\left(z_{1}, z_{2}\right) Y\left(z_{1}, z_{2}\right)=1,
\end{aligned}
$$

for all positive integers $n$ and $m$, this yields

$$
\begin{aligned}
& y[n, m]=\delta[n, m]+e^{-\lambda} y[n-1, m] \\
& \quad+\left(1-e^{-\lambda}\right) \sum_{k=0}^{n-1} \sum_{l=0}^{m} y[n-1-k, l] p_{X^{\prime}, Y^{\prime}}[k, m-l],
\end{aligned}
$$

where $\delta[n, m]=1$ when $n=0, m=0$ and $\delta[n, m]=0$ otherwise. Therefore, a direct pile-up correction procedure in the discrete case can be implemented as follows:

1) Compute a bi-dimensional histogram on $\llbracket 0, n_{0} \rrbracket \times \llbracket 0, m_{0} \rrbracket$ from $\left\{\left(X_{k}^{\prime}, Y_{k}^{\prime}\right), k=1 \ldots N\right\}$, as an estimate of $p_{X^{\prime}, Y^{\prime}}$

2) Compute recursively the terms $y[n, m]$ by means of $[6$,

3) Find the values of $k[n, m]$ by $(5)$,

4) Compute an estimate of the energy distribution, $\hat{p}_{Y}$, as

$$
\hat{p}_{Y}(m)=k\left[n_{0}, m\right]-k\left[n_{0}-1, m\right],
$$

where $n_{0}$ denotes the greatest bin of the histogram computed in the first step.
We emphasize that the presented algorithm has in common with the one presented in [4] the development of $y[n, m]$ by means of (6). However, there is a major difference between the two on the second step of the computation. Indeed, the algorithm presented in [4] computes multiple convolutions to compute $k[n, m]$, which increases the global complexity of the algorithm. On the other hand, the recursion in (5) reduces the computational load. This gain is crucial in practice, since the algorithm in [4] can be only used off-line, whereas our method can be theoretically implemented on real time apparatus. Moreover, the algorithm presented in [4] involves an expansion of the LHS of (3) in power series and its truncation up to an order fixed by the user. This yields an additional approximation error, which does not exist in the proposed approach. Eventually, when compared to [1], it can be noted that the presented approach does not include any numerical inversion of moment generating functions, since all the computations are performed in the time domain, making it less sensitive to numerical instability.

\section{ApPlicAtions}

We present results on simulations and real data. The pile-up durations and energies are obtained from the ADONIS digital instrumentation detailed in [6], [8].

\section{A. Results based on simulations}

In our experiments, we consider bi-dimensional histograms made with 16 bins for the durations and 256 bins for the energies. The durations of individual pulses are drawn according to a discretized Gamma distribution with a shape parameter of 4 and a scale parameter of 1 . The energies of individual pulses are drawn according to a discretized mixture of 6 normal distributions (representing the energy spectrum spikes) and a translated gamma density function (simulating the Compton continuum), more precisely $f_{Y} \propto 0.5 g+10 \mathcal{N}(40,1)+$ $10 \mathcal{N}(112,1)+\mathcal{N}(50,2)+\mathcal{N}(63,1)+2 \mathcal{N}(140,1)+\mathcal{N}(200,1)$, where $g(x) \stackrel{\text { def }}{=}(0.5+x / 200) e^{-(0.5+x / 200)}$ and $\mathcal{N}(x, y)$ is the Gaussian density function with mean $x$ and variance $y$. We compare the presented method with the algorithm developed in [4].Table I] summaries the execution times for a given identical approximation error, and the integrated squared error (ISE) for similar execution times. Numerical experiments were performed on an HP workstation with $2 \mathrm{GHz}$ Pentium processor, and the algorithms were coded in $\mathrm{C}$ language.

The results obtained by the proposed pile-up correction method are presented in Figure 3

\section{B. Results on real data}

We now present an application of the pile-up correction algorithm on a more complex source, namely a mixture of Americium 241 and Europium 152. The main peak locations in the energy spectra of these radionuclides are given in Table II . The combination of both elements results in closely piledup and real peaks (e.g. the piled-up peak at $119.08 \mathrm{keV}$ of Americium and the real peak at $121.78 \mathrm{keV}$ of Europium), illustrating the difficulty of the pile-up correction task. Results are shown in Figure 4 


\begin{tabular}{ccccc}
\hline & \multicolumn{2}{c}{ Proposed algorithm } & \multicolumn{2}{c}{ Algorithm from [4] } \\
\cline { 2 - 5 } Histogram number of bins (duration $\times$ energy) & Elapsed time & ISE & Elapsed time & ISE \\
\hline $16 \times 256$ & 0.031 & 0.1163941 & 0.265 & 0.1163942 \\
$16 \times 1024$ & 0.75 & 0.0926780 & 5.532 & 0.0926783 \\
$16 \times 4096$ & 11.498 & 0.0828218 & 93.75 & 0.0828220 \\
$16 \times 16384$ & 196.5 & 0.0815275 & 1586.73 & 0.0815278 \\
$32 \times 1024$ & 2.982 & 0.0814096 & 30.625 & 0.0814098 \\
$64 \times 1024$ & 2.9 & 0.0814335 & 70.327 & 0.0814338 \\
\hline
\end{tabular}

TABLE I: Comparison of discrete time pile-up correction techniques execution times and ISE.

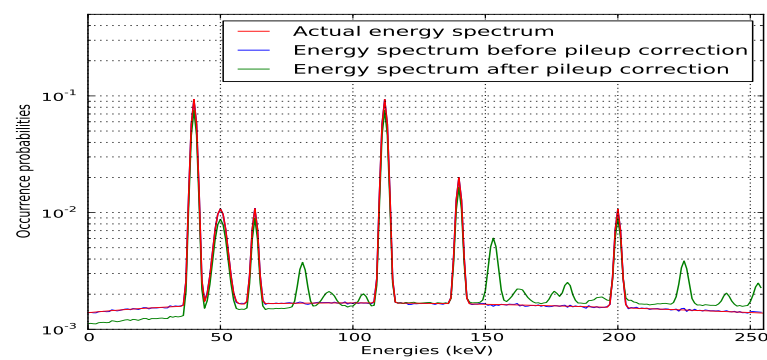

Fig. 3: Simulation results. The ideal and estimated energy spectra are almost superimposed.

\begin{tabular}{ccccc}
\hline \multicolumn{2}{c}{ Americium 241} & & \multicolumn{2}{c}{ Europium 152} \\
\cline { 1 - 2 } Energies $(\mathrm{keV})$ & Intensity $(\%)$ & & Energies $(\mathrm{keV})$ & Intensity $(\%)$ \\
26.34 & 2.31 & & 121.78 & 28.41 \\
33.20 & 0.12 & & 244.70 & 7.55 \\
59.54 & 35.92 & & 295.94 & 0.44 \\
102.98 & 0.0195 & & 329.43 & 0.13 \\
& & & 344.28 & 26.59 \\
& & & 367.79 & 0.86 \\
\hline
\end{tabular}

TABLE II: Main peaks in the gamma energy spectra of Americium 241 and Europium 152 between 0 and $400 \mathrm{keV}$.

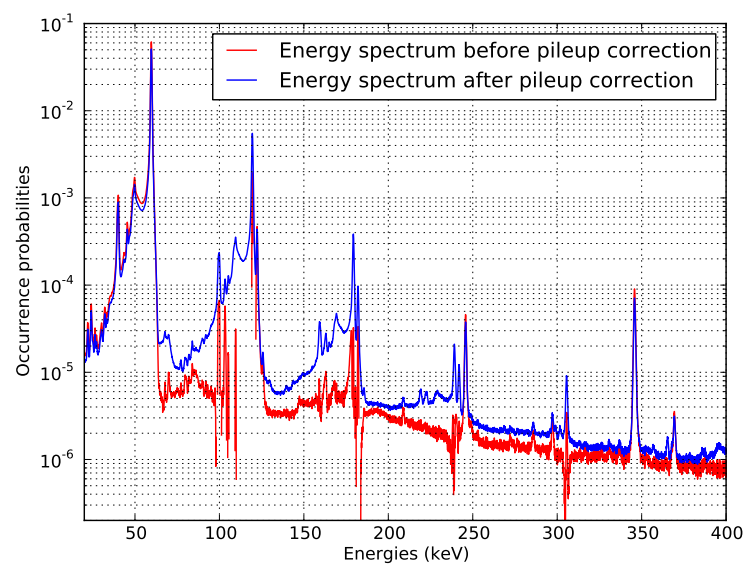

Fig. 4: pile-up correction on a complex mixture, with total activity $\lambda=280000$ photons per second (zoom from 0 to 400 $\mathrm{keV})$.

\section{Discussion}

As seen in the results of the simulations, the pile-up effect on simulations is perfectly corrected: all the fake spikes caused by pulse overlapping are discarded, and the obtained estimation of the energy spectrum almost perfectly matches the objective density. It is even more remarkable to observe that the Gamma function representing the Compton diffusion effect is also well corrected. This is done with a valuable gain in terms of calculation times, as expected. The gain of precision is more marginal, however a slight improvement can be observed for all the cases. Note that even this slight improvement is important in practice, since in our applications the energy spectra are commonly displayed in logarithmic scale. As shown, the presented method is close to ten times faster than [4], without sacrificing precision.

The results displayed in Figure 4 are interesting to detail in light of Table II We can see that the Compton background is well corrected, and that numerous fake peaks are discarded, even when they are located close to actual peaks. For example, the Compton background is corrected in [100 keV-150 keV] and $[200 \mathrm{keV}-250 \mathrm{keV}]$ regions while preserving the 244.7 $\mathrm{keV}$ Europium peak and revealing the peak at $121.8 \mathrm{keV}$. The discontinuities obtained are due to the fact that no positivity constraint is assumed on the resulting histogram coefficients, so that the logarithmic value cannot always be computed. We also remark that the correction of the Compton continuum makes some actual, but less significant, peaks appear more clearly, e.g. Europium peaks at $212 \mathrm{keV}$ and $285 \mathrm{keV}$.

In Figure 4, we observe that our pile-up correction method, though efficient, is not as efficient as expected from the simulated data in Figure 3 . Indeed, one can see small deformations of the Compton continuum located at peaks positions, and we can notice that some fake peaks (e.g. at $119.08 \mathrm{keV}$ ) are only partially corrected, and that an artifact peak with lesser variance remains. This behavior is explained by the fact that observed busy discrete energies $Y_{n}^{\prime}$ are corrupted by an additive noise due to the instrumental system, which is absent from the model. Neglecting the additive remains plausible in the case of detectors with high signal-noise ratio, such as high purity Germanium detectors. Nevertheless, further investigations must be done in order to adapt this approach to a wider class of detectors.

\section{CONCLUSION}

We presented an adaptation of the pile-up correction formula, fitted for digital instrumentation and digital spectrometric signals. This led to a fast algorithm for pile-up correction. Results on simulations and real data show that the proposed method corrects efficiently the distortion in energy spectra caused by the pile-up effect. 


\section{REFERENCES}

[1] T. Trigano, A. Souloumiac, T. Montagu, F. Roueff, and E. Moulines, "Statistical Pileup Correction Method for HPGe Detectors," IEEE Transactions on Signal Processing, vol. 55, no. 10, pp. 4871-4881, 2007.

[2] R. Novak and M. Vencelj, "Gauss-Seidel iterative method as a RealTime pileup solver of scintillator pulses," IEEE Transactions in Nuclear Science, vol. 56, no. 6, pp. 3680-3687, 2009.

[3] M. Bolic, V. Drndarevic, and W. Gueaieb, "Pileup correction algorithms for Very-High-Count-Rate Gamma-Ray spectrometry with $\mathrm{NaI}(\mathrm{Tl})$ detectors," IEEE Transactions on Instrumentation and Measurement, vol. 59, no. 1, pp. 122-130, Jan. 2010.

[4] T. Trigano, T. Dautremer, E. Barat, and A. Souloumiac, "Pile-up correction algorithms for nuclear spectrometry," in IEEE International Conference on Acoustics, Speech, and Signal Processing, vol. 4, no. iv, march 2005, pp. 441-444.

[5] T. Trigano, F. Roueff, E. Moulines, and A. Souloumiac, "Nonparametric Inference of Photon Energy from Indirect Measurements," Bernoulli, vol. 13, no. 2, pp. 365-388, 2007.

[6] E. Barat, T. Dautremer, T. Montagu, and S. Normand, "A new concept of X/gamma pulse analyzer," in Advancements in Nuclear Instrumentation Measurements Methods and their Applications, vol. N250, 2009.

[7] T. Trigano, "Statistical Signal Processing in Nuclear Spectrometry: an Application to Pileup Correction in Gamma Spectrometry," Ph.D. dissertation, ENST, 2005.

[8] E. Barat, T. Dautremer, T. Montagu, and J.-C. Trama, "A Bimodal Kalman Smoother for Nuclear Spectrometry," Nuclear Instruments and Methods in Physics Research Section A: Accelerators, Spectrometers, Detectors and Associated Equipment, vol. 567, pp. 350-352, 2006.

\section{APPENDIX}

\section{PROOF OF (2)}

This section describes the proof of Equation (2). We assume that $\lambda$ is known. The proof is close to the continuous case, described in [5]. We introduce a similar auxiliary function, based upon the probability that we are standing in an idle period at time $m$, and that the total accumulated energy before this time is equal to $n$ :

$$
\rho[m, n] \stackrel{\text { def }}{=} \mathbb{P}\left(s[m]=0 \cap \sum_{j=1}^{m-1} s[j]=n\right),(m, n) \in \mathbb{N}^{2} .
$$

We also define

$$
R(s, p) \stackrel{\text { def }}{=} \sum_{m \geq 0} \sum_{n \geq 0} \rho[m, n] s^{m} p^{n}
$$

as the moment generating function associated to $\rho$. The follows proposition computes $R$ using the properties of homogeneous Poisson processes.

Proposition A.1: Under previous assumptions, for any $(s, p) \in \mathbb{C}^{2}$ belonging to the definition domain of $R$,

$$
\begin{array}{r}
R\left(z_{1}, z_{2}\right)=\sum_{n \geq 0}\left(\exp \left(\lambda n-\lambda \sum_{m=0}^{+\infty} k[n, m] z_{2}^{m}\right)\right) z_{1}^{n} \\
-\frac{1}{1-\mathrm{e}^{-\lambda} z_{1}} .
\end{array}
$$

Proof: Denote by $\left\{\mathcal{N}_{t}, t \geq 0\right\}$ the counting process associated to the homogeneous Poisson process $\left\{T_{k}, k \geq 0\right\}$ of the arrivals, more explicitly $\mathcal{N}_{t}=\sum_{n=1}^{\infty} 1\left\{T_{n} \leq t\right\}$. We obtain by conditioning that:

$$
\begin{aligned}
& \rho[m, n]=\mathrm{e}^{-\lambda m}+\sum_{k \geq 1} \mathbb{P}\left(\mathcal{N}_{m}=k\right) \\
& \times \mathbb{P}\left(\left\{T_{i}+X_{i} \leq m\right\}_{i=1}^{n}, \sum_{l=1}^{k} Y_{l}=n \mid \mathcal{N}_{m}=k\right) .
\end{aligned}
$$

The conditional distribution of the arrival times $\left(T_{1}, \ldots, T_{k}\right)$ given $\left\{\mathcal{N}_{m}=k\right\}$ is equal to the distribution of the order statistics of $n$ independent and identically distributed uniform random variables on $[0, m]$; hence, for any $n$-tuple $\left(m_{1}, \ldots, m_{k}\right)$ of positive real numbers,

$$
\begin{aligned}
\mathbb{P}\left(T_{1} \leq m_{1}, \ldots, T_{k} \leq m_{k} \mid \mathcal{N}_{m}=k\right) & \\
& =\mathbb{P}\left(U_{(1)} \leq m_{1}, \ldots, U_{(k)} \leq m_{k}\right),
\end{aligned}
$$

where $\left\{U_{l}\right\}_{l=1}^{k}$ are independent and identically distributed random variables uniformly distributed on $[0, m]$ and $U_{(1)} \leq$ $\cdots \leq U_{(k)}$ are the order statistics of this sample. Therefore, (8) and (9) imply that

$$
\begin{aligned}
& A \stackrel{\text { def }}{=} \mathbb{P}\left(\left\{T_{i}+X_{i} \leq m\right\}_{i=1}^{k}, \sum_{l=1}^{k} Y_{l}=n \mid \mathcal{N}_{m}=k\right) \\
& =\frac{1}{m^{k}} \int \cdots \int \prod_{l=1}^{k} \mathbf{1}\left\{u_{l}+x_{l} \leq m\right\} \mathbf{1}\left\{\sum_{l=1}^{k} y_{l}=n\right\} \prod_{l=1}^{k} p_{X, Y}\left(d x_{l}, d y_{l}\right) d l
\end{aligned}
$$

Using Fubini's theorem, we find that

$$
A=\frac{1}{m^{k}} \int \cdots \int \mathbf{1}\left\{\sum_{l=1}^{k} y_{l}=n\right\} \prod_{l=1}^{k} \kappa\left(m, y_{l}\right),
$$

where $\kappa(m, n)$ is the probability kernel defined by

$$
\kappa(m, n) \stackrel{\text { def }}{=} \sum_{k \geq 0}(m-k) \mathbf{1}\{k \leq m\} p_{X, Y}(k, n) .
$$

Eventually, computing the moment generating function leads to

$$
\begin{aligned}
& R\left(z_{1}, z_{2}\right) \stackrel{\text { def }}{=} \sum_{m \geq 0} \sum_{n \geq 0} \rho[m, n] z_{1}^{m} z_{2}^{n} \\
& =\sum_{m \geq 0}\left(\sum_{n \geq 0} \rho[m, n] z_{2}^{n}\right) z_{1}^{m} \\
& =\sum_{m \geq 0}\left(\sum_{n \geq 1} \frac{\lambda^{n}}{n !} \mathrm{e}^{-\lambda m}\left[\sum_{j \geq 1} k[m, j] z_{2}^{j}\right]\right) z_{1}^{m} \\
& =\sum_{m \geq 0}\left(\mathrm{e}^{-\lambda m+\lambda \sum_{j \geq 1} k[m, j] z_{2}^{j}}-\mathrm{e}^{-\lambda m}\right) z_{1}^{m} \\
& =\sum_{m \geq 0}\left(\mathrm{e}^{-\lambda m+\lambda \sum_{j \geq 1} k[m, j] z_{2}^{j}}\right) z_{1}^{m}-\frac{1}{1-\mathrm{e}^{-\lambda} z_{1}},
\end{aligned}
$$

which completes the proof.

On the other hand, the moment generating function of $\rho$ can also be computed using the fact that the observed process is a marked renewal process, with renewal distribution $p_{X^{\prime}} * g$, where $p_{X^{\prime}}$ is the distribution of $X^{\prime}$, and $g$ is the geometric distribution with parameter $\mathrm{e}^{-\lambda}$. Therefore, the following proposition holds:

Proposition A.2: Under the same assumptions and notations, we have its definition domain:

$$
\begin{aligned}
& \left(1-\mathrm{e}^{-\lambda}\right) s R\left(z_{1}, z_{2}\right)=\frac{\left(1-\mathrm{e}^{-\lambda}\right) z_{1}}{1-z_{1} \mathrm{e}^{-\lambda}-\left(1-\mathrm{e}^{-\lambda}\right) z_{1} P_{X^{\prime}, Y^{\prime}}\left(z_{1}, z_{2}\right)} \\
& -\frac{\left(1-\mathrm{e}^{-\lambda}\right) z_{1}}{1-z_{1} \mathrm{e}^{-\lambda}}
\end{aligned}
$$


Proof: The proof is based on classical renewal arguments and the fact that for all integer $k$, the idle period $Z_{k} \stackrel{\text { def }}{=}$ $\#\left\{T_{k-1}^{\prime} \leq m \leq T_{k}^{\prime} ; s[m]=0\right\}$ is distributed according to a geometric distribution with parameter $\mathrm{e}^{-\lambda}$. Observe that for all $m \geq 2$ and for all integer $n$, the event $\{s[m-1]=0, s[m]>$ $\left.0, \sum_{k=1}^{m-1} s[k]=n\right\}$ can be decomposed as follows:

$$
\begin{aligned}
& \left\{s[m-1]=0, s[m]>0, \sum_{k=1}^{m-1} s[k]=n\right\} \\
& =\bigcup_{l \geq 2}\left\{T_{l}^{\prime}=m, \sum_{k=1}^{l-1} Y_{k}^{\prime}=n\right\} \\
& =\bigcup_{l \geq 2}\left\{\sum_{k=1}^{l-1}\left(Z_{k}+X_{k}^{\prime}\right)+Z_{l}=m, \sum_{k=1}^{l-1} Y_{k}^{\prime}=n\right\} .
\end{aligned}
$$

Since $\mathbb{P}\left(s[m-1]=0, s[m]>0, \sum_{k=1}^{m-1} s[k]=n\right)=(1-$ $\left.\mathrm{e}^{-\lambda}\right) \rho[m-1, n]$, equation $[13$ leads to

$$
\begin{aligned}
(1- & \left.\mathrm{e}^{-\lambda}\right) \rho[m-1, n] \\
& =\sum_{l \geq 2} \mathbb{P}\left(\sum_{k=1}^{l-1}\left(Z_{k}+X_{k}^{\prime}\right)+Z_{l}=m, \sum_{k=1}^{l-1} Y_{k}^{\prime}=n\right) .
\end{aligned}
$$

Due to independence of $Z_{l}$ with $\left\{X_{k}^{\prime}+Z_{k}, k=1 \ldots l-1\right\}$ and of $\left\{X_{k}^{\prime}, k \geq 1\right\}$ with $\left\{Z_{k}, k \geq 1\right\}$, taking the moment generating function of the latter equation yields to

$$
\left(1-e^{-\lambda}\right) z_{1} R\left(z_{1}, z_{2}\right)=G\left(z_{1}\right) \sum_{l \geq 1}\left(G\left(z_{1}\right) P_{X^{\prime}, Y^{\prime}}\left(z_{1}, z_{2}\right)\right)^{l},
$$

where $G\left(z_{1}\right)$ is the moment generating function of a geometric distribution, that is:

$$
G\left(z_{1}\right) \stackrel{\text { def }}{=} \frac{\left(1-\mathrm{e}^{-\lambda}\right) z_{1}}{1-\mathrm{e}^{-\lambda} z_{1}} .
$$

Thus, we get from (14) that

$$
\begin{aligned}
& \left(1-e^{-\lambda}\right) z_{1} R\left(z_{1}, z_{2}\right) \\
& =\frac{\left(1-\mathrm{e}^{-\lambda}\right) z_{1}}{1-\mathrm{e}^{-\lambda} z_{1}} \times\left(\frac{1}{1-\frac{\left(1-\mathrm{e}^{-\lambda}\right) z_{1}}{1-\mathrm{e}^{-\lambda} z_{1}} P_{X^{\prime}, Y^{\prime}}\left(z_{1}, z_{2}\right)}-1\right) \\
& =\frac{\left(1-\mathrm{e}^{-\lambda}\right) z_{1}}{1-z_{1} \mathrm{e}^{-\lambda}-\left(1-\mathrm{e}^{-\lambda}\right) z_{1} P_{X^{\prime}, Y^{\prime}}\left(z_{1}, z_{2}\right)}-\frac{\left(1-\mathrm{e}^{-\lambda}\right) z_{1}}{1-z_{1} \mathrm{e}^{-\lambda}},
\end{aligned}
$$

which ends the proof.

Equation (2) is therefore a straightforward consequence of Propositions A.1 and A.2. 